Published in final edited form as:

Trends Cancer. 2019 May ; 5(5): 283-296. doi:10.1016/j.trecan.2019.02.010.

\title{
YAP/TAZ Signaling and Resistance to Cancer Therapy
}

\author{
Chan Nguyen ${ }^{1}$ and Chunling $\mathrm{Yi}^{1,2}$ \\ ${ }^{1}$ Lombardi Comprehensive Cancer Center, Georgetown University Medical Center, Washington, \\ DC, USA;
}

\begin{abstract}
Drug resistance is a major challenge in cancer treatment. Emerging evidence indicates that deregulation of YAP/TAZ signaling may be a major mechanism of intrinsic and acquired resistance to various targeted and chemotherapies. Moreover, YAP/TAZ-mediated expression of PD-L1 and multiple cytokines is pivotal for tumor immune evasion. While direct inhibitors of YAP/TAZ are still under development, FDA-approved drugs that indirectly block YAP/TAZ activation or critical downstream targets of YAP/TAZ have shown promise in the clinic in reducing therapy resistance. Finally, BET inhibitors, which reportedly block YAP/TAZ-mediated transcription, present another potential venue to overcome YAP/TAZ-induced drug resistance.
\end{abstract}

\section{Keywords \\ Hippo pathway; YAP; TAZ; Cancer therapy resistance \\ CANCER THERAPY RESISTANCE AND REGULATION OF YAP/TAZ SIGNALING}

Despite the dramatic improvement in treatment outcomes for several highly aggressive cancer types in the recent decade, complete and sustained response remains rare in most malignancies due to development of primary or acquired resistance [1]. It is thus essential to identify mechanisms of resistance to design optimal treatment strategies to prevent or overcome relapse. Emerging literature indicates that the Hippo-YAP/TAZ pathway might play a central role in mediating resistance to major classes of cancer therapeutics.

The Hippo pathway was originally discovered in Drosophila as a major developmental pathway that controls organ size and later found to be conserved in mammals [2-5]. The core mammalian Hippo pathway consists of a kinase cascade in which MST1/2 kinases (orthologs of Hippo in Drosophila), facilitated by scaffold proteins such as SAV1 and NF2/ MERLIN, phosphorylate and activate LATS1/2 kinases and the regulatory protein MOB1 [2,5,6]. Activated LATS1/2 in turn phosphorylate YAP and TAZ, priming binding to 14-3-3

\footnotetext{
${ }^{2}$ Corresponding author. cy232@georgetown.edu.

Publisher's Disclaimer: This is a PDF file of an unedited manuscript that has been accepted for publication. As a service to our customers we are providing this early version of the manuscript. The manuscript will undergo copyediting, typesetting, and review of the resulting proof before it is published in its final citable form. Please note that during the production process errors may be discovered which could affect the content, and all legal disclaimers that apply to the journal pertain.
} 
and $\beta-\operatorname{TrCP}$, which mediate cytoplasmic retention and proteasomal degradation, respectively. Parallel or upstream of MST1/2, the MAP4K family of Ste20-like kinases and TAOK1-3 can also directly or indirectly phosphorylate and activate LATS1/2, thereby inhibiting YAP/TAZ activity [7]. Additionally, many cellular processes such as mechanotransduction and various metabolic pathways have been shown to regulate YAP/TAZ nuclear translocation independent of the core Hippo kinases [8-13]. Once inside the nucleus, YAP/TAZ function as co-activators to multiple transcription factors including TEAD1-4, RUNX2, p73, etc [14-17]. In particular, the TEA-Domain family transcription factors, TEAD1-4, share significant overlap with YAP/TAZ in genomic binding sites and are primary partners of YAP/TAZ in transcribing genes that regulate growth, survival, inflammation, angiogenesis, mechanotransduction, oxidative stress response and stemness[16-18].

A growing body of literature implicates YAP/TAZ activation in resistance to targeted therapies, chemotherapy, radiation, and also, potentially immunotherapies. Though detailed mechanisms vary, they converge on YAP/TAZ-mediated transcription, either through inactivation of upstream YAP/TAZ negative regulators or by direct activation of YAP and/or TAZ. Here, we provide a brief overview of numerous reported YAP/TAZ-dependent mechanisms that lead to therapy resistance. We also address caveats and gaps in existing literature and offer our perspective on potential strategies to overcome YAP/TAZ-mediated resistance to cancer therapy.

\section{YAP/TAZ SIGNALING AND RESISTANCE TO TARGETED THERAPY}

Targeted therapies inhibit specific molecules and pathways that are crucial for growth and survival of cancer cells. Effective targeted therapy in cancer was first demonstrated with inhibition of Bcr-Abl, a product of the Philadelphia chromosome fusion oncogene, with Imatinib in Chronic Myelogenous Leukemia (CML) and Acute Lymphocytic Leukemia (ALL), which resulted in objective and durable response in more than $50 \%$ of patients $[19,20]$. Other dependencies in tumors have been also exploited, including EGFR in EGFR mutant non-small-cell lung cancer (NSCLC) with cetuximab, erlotinib; VEGFR in VHL mutant clear cell renal cell carcinoma (ccRCC) with bevacizumab, axitinib; BRAF (vemurafenib, dabrafenib) or MEK in BRAF-mutant melanoma with vemurafenib, dabrafenib or with trametinib, cobimetinib, repectively; or estrogen receptor (ER) in $\mathrm{ER}^{+}$ breast cancer with tamoxifen, fulvestrant [21]. However, most patients develop primary or acquired resistance to targeted therapies. Deregulation of Hippo signaling and subsequent activation of YAP/TAZ is a major resistance mechanism to multiple targeted therapies, particularly against EGFR and MAPK inhibitors (MAPKi). Below, we will discuss how dysregulation of Hippo-YAP/TAZ signaling helps tumor cells bypass pathway inhibition by activation of alternative survival pathways or antiapoptotic signaling (Figure 1).

Approximately half of all melanoma tumors harbor activating mutation in the BRAF gene, which leads to constitutive activation of MAPK (MEK-ERK) signaling [22]. Combined therapy with BRAF and MEK inhibitors can lead to rapid tumor regression and significant long-term benefit in patients with metastatic $\mathrm{BRAF}^{\mathrm{V} 600 \mathrm{E}}$ melanoma, although development of acquired resistance remains a formidable obstacle [23-25]. In an effort to identify 
mechanisms of resistance to BRAF inhibitor (BRAFi), a pooled genome-scale CRISPR knockout screen in human melanoma cells identified NF2 sgRNAs among the most enriched after BRAFi treatment [26]. NF2 encodes Merlin, a member of the ERM protein family that functions as a scaffold bridging cytoskeletal dynamics with activation of the MST1/2LATS1/2 kinase cascade, negatively regulating YAP/TAZ activity [27,28]. Another genomescale CRISPR activation screen targeting >10,000 long-noncoding RNA (lncRNA) identified IncRNA EMICERI as a mediator of BRAFi resistance [29]. Intriguingly, it showed that EMICERI confers resistance to BRAFi resistance by promoting the expression of its genomic neighbor MOB3B - a component of the STRIPAK (striatin-interacting phosphatase and kinase) complex that inhibits MST1/2 and LATS1/2 [29-31]. Finally, genome-wide shRNA screens confirmed that YAP mediates resistance to BRAFi and MAPKi in BRAF mutant cancer cells [32-34]. Interestingly, BRAFi treatment was shown to induce actin cytoskeleton remodeling, which induces activation of YAP via Rho-mediated LATS1/2 inhibition in BRAF-mutant melanoma cells [33,34].

The RAS family of small GTPases - KRAS, HRAS and NRAS, which directly activate the MAPK and other pathways are also frequently mutated in cancer [35]. While RAS inhibitors are still under preclinical development, genetic studies were carried out to identify potential bypass mechanisms of oncogenic Ras ablation. Studies assessing tumor relapse following extinction of oncogenic Kras in a genetically engineered mouse model of advanced Pancreatic Ductal Adenocarcinoma (PDAC), and detected amplification of the Yap gene locus in three out of eight relapsed tumors analyzed [36]. Independently, in genome-scale cDNA screens, Yap was the top hit in promoting survival upon KRAS knockdown in a human KRAS mutant colon cancer cell lines [37]. Finally, a recent study showed that YAP ablation was sufficient to overcome resistance of Kras mutant lung cancer cells to the combined treatment with Selumetinib, a MEK inhibitor, and Momelotinib, a TBK/JAK inhibitor [38]. Together, these studies establish YAP as a key driver of escape from KRAS/ MAPK dependency.

Several studies explored the mechanisms underlying YAP-mediated resistance to blockade of KRAS-MAPK signaling. While Yap was shown to cooperate with Tead2 and E2F transcription factors to promote cell cycle progression in Kras-ablated PDAC cells [36], it was reported to rescue the loss of oncogenic KRAS in human lung cancer cells by partnering with AP-1 transcription factor FOS to promote epithelial-mesenchymal transition (EMT) independent of TEADs [37]. In response to treatment with MAPKi, YAP was found to prevent apoptosis in BRAF or KRAS mutant tumor cells through TEAD-mediated transcription of BCL2L1, which encodes anti-apoptotic protein Bcl-xL[32]. Yet according to another study, depletion of YAP sensitizes KRAS mutant PDAC cells to a pan-RAF inhibitor LY3009120 by blocking the compensatory activation of PI3K-AKT signaling [39]. These findings indicate that Yap likely induces therapy resistance through multiple downstream effectors, and targeting one particular Yap-mediated bypass mechanism will unlikely be sufficient to overcome resistance to KRASMAPK pathway blockade. For example, while combination treatment of BRAF/MEK and PI3K inhibition demonstrated remarkable results in preclinical models of Kras-mutant NSCLC[40-42], clinical trials were plagued with heterogeneous response [43-45], in part due to reduction in ratio between pro-apoptotic $\mathrm{BIM}$ and anti-apoptotic Bcl-2 proteins, resulting in reduced apoptotic propensity[46]. Thus, 
it would be important in further studies to determine the relative contributions of various Yap effector pathways to the overall resistance development under different contexts and how differences in mutation profiles, epigenetic states and tumor microenvironment may cause subpopulations of tumor cells to activate distinct bypass mechanisms to the same treatment.

Upstream of RAS-MAPK signaling, receptor tyrosine kinases (RTKs) such as EGFR are also frequently mutated or amplified to drive tumorigenesis and drug resistance [47,48]. Monoclonal antibodies or small-molecule tyrosine kinase inhibitors (TKI) have shown efficacy in patients with RTK-addicted tumors, although resistance develops in most patients [21]. Numerous studies linked activation of YAP/TAZ to TKI resistance, including first and second generations of EGFR inhibitors (EGFRi) and the multi-kinase TKI sorafenib [4960]. The precise mechanisms by which YAP/TAZ drive TKI resistance remain poorly understood. However, given that RTK functions directly upstream of the MAPK pathway, it is likely that that a similar set of YAP/TAZ transcriptional targets confer resistance to both TKI and MAPK pathway inhibitors. Indeed, AXL, a direct transcriptional target of YAP/ TAZ, mediates resistance to both BRAFi and EGFRi [49,50,55,61-63]. Besides AXL, other YAP/TAZ targets linked to RTKi inhibition include CTGF, EGFR, ERBB3, EGFR and EMT genes $[55,56,58,64-66]$.

Other targeted therapies with resistance mechanisms associated with Hippo signaling include mTOR, TBK/IGF1R, imatinib, CDK4/6, WEE1 and Aurora-A inhibitors [38,6771]. Taken together, these studies provide concrete evidence for the involvement of HippoYAP/TAZ signaling in development of resistance to targeted therapies across multiple malignancies.

\section{YAP/TAZ SIGNALING AND RESISTANCE TO CHEMOTHERAPY}

Chemotherapy encompasses a broad range of drugs that non-specifically inhibit cell division. There are multiple subtypes of chemotherapy, including anti-microtubule, antimetabolites, and DNA damage agents, all designed to cause damage to highly proliferative cells. A vast body of literature links Hippo-YAP/TAZ signaling to resistance to chemotherapy in numerous solid cancers. Below, we will summarize the known upstream mechanisms that activate YAP/TAZ in response to cytotoxic agents and how notable YAP/TAZ targets enable tumor cells to overcome the detrimental effects of chemotherapy drugs (Figure 2).

\section{Anti-Microtubule Drugs}

Anti-microtubule compounds block mitosis and induce apoptosis by disrupting the assembly or disassembly of microtubules. Taxane (Paclitaxel, Docetaxel) is a major class of antimicrotubule drugs widely used in treatment of ovarian, lung, breast, cervical and prostate cancer and acts to stabilize GDP-bound tubulin, thereby preventing microtubule disassembly during cell cycle $[72,73]$. Resistance to taxane has been associated with deregulation of upstream YAP/TAZ regulators involving various mechanism, particularly microRNAs (miRNAs) [64,74-84]. miRNA-363, which is upregulated in taxane-resistant ovarian cancer cells, confers resistance by directly targeting LATS2 mRNA translation, which in turn activates YAP [81]. miR-181c is upregulated in PDAC and promotes taxane resistance by 
directly suppressing the translation of multiple Hippo pathway components including MST1, LATS2, MOB1 and SAV1 [85]. Activation of Protease-activated receptor-1 (PAR1), a Gprotein coupled receptor (GPCR), induces stemness and multi-drug chemoresistance in gastric cancer cells by promoting YAP dephosphorylation and nuclear translocation through Rho-mediated inactivation of LAST1/2 [34,83]. Conversely, Cyclin-dependent kinase 1 (CDK1), sensitizes ovarian cancer cells to taxanes by phosphorylating LATS1/2 and YAP/TAZ [75,78,80,84,86,87]. On one hand, CDK1-mediated phosphorylation of LATS1/2 does not appear to interfere with their ability to phosphorylate YAP/TAZ, but rather cause LATS1/2 to associate with PIN1, a peptidyl-prolyl cis/trans isomerase (PPIase), and promotes drug sensitivity through yet-to-be-defined mechanisms [80]. On the other hand, following taxane treatment, CDK1 directly phosphorylates YAP/TAZ at sites distinct from those targeted by LATS1/2, reducing TAZ protein stability while suppressing YAP transcriptional activity by reducing its interaction with TEADs $[75,84]$.

Multiple transcription targets may contribute to taxane resistance downstream of YAP/TAZ. Several studies suggest that YAP/TAZ are enriched in cancer cells (CSCs) and promote pluripotent transcription program resulting in increased tumor initiating ability and chemotherapy resistance $[15,77,82,83,88]$. In particular, the YAP/TAZ/TEAD transcription complexes induce expression of multidrug transporters $\mathrm{ABCB} 1$ and $\mathrm{ABCC} 1$ in ovarian and liver CSCs and potentially mediate PAR1-induced upregulation of ABCG2 in gastric CSCs $[82,83,88]$. Upregulation of multidrug transporters is expected to increase drug efflux. Indeed, overexpression of a constitutively active TAZ enhances the ability of breast cancer cells to exclude Hoescht dye, which could be reversed with the multidrug transporter inhibitor Verapamil [77]. Finally, YAP/TAZ may also indirectly promote taxane resistance through canonical transcriptional targets CTGF and CYR61, which inhibit taxane-induced apoptosis by upregulating anti-apoptotic proteins Bcl-xL, cIAP1 and Survivin while suppressing p53 via integrin-mediated activation of MAPK or NF- $\mathrm{BB}$ signaling [89-92].

\section{Anti-Metabolites}

Anti-metabolites represent another major class of cytotoxic drugs mainly composed of metabolite analogs designed to interfere with critical metabolic pathways necessary for cell proliferation and survival. Fluorouracil (5-FU) and gemcitabine, the respective nucleoside analogs of Uracil and Cytidine, are commonly used as first-line treatment in pancreatic, breast, ovarian and colorectal cancer with variable benefits and predictable relapse [93-99]. Antifolates such as methotrexate and pemetrexed are the other major class of antimetabolites that antagonize the actions of folic acid (Vitamin B9), whose derivatives fuel the folate cycle that supports serine, methionine, thymidine and purine de novo biosynthesis [100]. Hippo signaling was implicated in resistance to both types of anti-metabolite drugs $[64,83,85,101-110]$.

Similar to anti-microtubule resistance, multiple explorative studies linked miRNA-mediated deregulation of Hippo signaling to resistance to 5-FU and gemcitabine in cancer cells, including miR-181c and miR-455-3p in pancreatic cancer and miR-874-3p in colorectal cancer $[85,101,102,109]$. Downstream of YAP/TAZ, CTGF and CYR61 were also found to play major roles in anti-metabolite resistance through the activation of Integrin signaling 
[92,111]. Furthermore, several studies implicated YAP/TAZ as positive regulators of multidrug transporters (ABCs) $[82,83,88]$. However, contrary to these reports, a recent study found that expression of a constitutively active YAP or knockdown of MERLIN or LATS2 downregulates multidrug transporters such as ABCB4, ABCC3 and ABCG2 and reverts gemcitabine resistance induced by high cell density in pancreatic cancer cells [107]. This study differs from others in that most of the experiments were performed under high confluence conditions. Because no knockdown of YAP was done in low confluent cells when YAP is presumably active, it is difficult to assess whether the effects induced by forcing YAP activation in conditions where it is normally suppressed reflect the physiological function of YAP. Finally, YAP/TAZ were recently reported to induce the expression of high affinity glucose and AA transporters [112-116], which may indirectly blunt the effects of anti-metabolite drugs.

\section{DNA Damaging Agents}

DNA damaging agents refers to compounds that induce cell cycle arrest and apoptosis by either directly alkylating DNA (cisplatin, temolozomide, etc.) or inhibiting topoisomerases the enzymes that unwind the double-stranded DNA during replication and DNA repair (doxorubicin, etoposide). Hippo signaling was linked to resistance to DNA damaging agents in numerous studies [77,82,83,88,104,108,110,117-134]. While YAP/TAZ confer resistance to DNA damaging agents through a similar set of transcriptional targets as to other chemo drugs $[66,77,82,89,92,118,119,127,128,135,136]$, distinct upstream mechanisms appear to underlie YAP/TAZ activation. Three studies reported downregulation of MST1 following doxorubicin or cisplatin treatment either due to Hsp70-mediated degradation or translation blockade caused by miR-149-5p, which also targets the scaffolding protein SAV1 $[110,123,129]$. In addition, AJUBA, a negatively regulator of LATS1/2, is upregulated along with YAP/TAZ in cisplatin-resistant cervical cancer cells [132]. Finally, Hippo-independent mechanisms were also reported to contribute to YAP activation. Doxorubicin-induced increase in YAP expression was linked to downregulation of miR-590-5p, which directly inhibits YAP translation [88]. Furthermore, SIRT1-mediated-deacetylation of YAP increases its nuclear translocation and interaction with TEAD following cisplatin treatment [122]. Similarly, overexpression of an acetylation-resistant YAP mutant modestly boosted resistance to another DNA-alkylating agent methyl methanesulfonate (MMS), although YAP acetylation driven by $\mathrm{CBP} / \mathrm{p} 300$ was increased in response to MMS and did not affect YAP nuclear translocation [137].

\section{YAP/TAZ SIGNALING AND RESISTANCE TO IMMUNE THERAPY}

Immune evasion presents a major challenge but also an opportunity in cancer treatment. Most notably, PD-L1 and CTLA-4 are cell surface markers often expressed on tumor cells that bind to their cognate receptors on cytotoxic $\mathrm{T}$ cells (CTLs), inducing $\mathrm{T}$ cell exhaustion and preventing CTL-mediated tumor-specific killing. Monoclonal antibodies targeting PDL1/CTLA-4 (Artezolizumab, Ipilimumab, etc.) or PD-L1 receptor PD-1 (Nivolumab, Pembrolizumab.) are successfully used to reactivate $\mathrm{T}$ cells in certain tumors, although they fail to work in most tumors due to the existence of additional immune suppressive 
mechanisms [138-140]. Here, we will explore the potential roles of Hippo-YAP/TAZ signaling in modulating cancer immunotherapy response (Figure 3).

Multiple studies identified PD-L1 as a direct transcriptional target of YAP/TAZ/TEAD and showed that YAP and/or TAZ activation upregulates PD-L1 in human breast cancer, NSCLC, mesothelioma and melanoma cells, although it was reported that the TEAD binding sites are not conserved on the mouse PD-L1 promoter [141-146]. Besides promoting PD-L1 expression, YAP may also suppress $\mathrm{T}$ cell function by inducing the expression of cytokines that drive polarization and recruitment of myeloid-derived suppressing cells (MDSCs) and tumor-associated macrophages (TAMs), which inactivate $\mathrm{T}$ cells and induce $\mathrm{T}$ cell apoptosis through multiple mechanisms [147]. Yap promotes the recruitment of MDSCs in syngeneic prostate cancer models by directly transcribing Cxcl5, which attracts CXCR2-expressing MDSCs to the tumor microenvironment [148]. Yap also drives the recruitment and systematic polarization of MDSCs by promoting the expression of Il-6, Csf1-3, Tnfa, Il-3, Cxcl1/2, and Ccl2 in genetically engineered PDAC models [149]. Yap-induced tumor secretome is also responsible for recruitment of TAMs in pancreatic and liver tumor models [149-151].

\section{CONCLUDING REMARKS}

In summary, YAP/TAZ signaling is a pivotal regulator of response to targeted, chemo, and immune therapies in many types of solid tumors. However, there are currently no clinically viable drugs that directly target this pathway. Verteporfin(VP), a photosensitizing medication used to treat abnormal blood vessels in the eye, was the first compound identified to directly inhibit YAP-TEAD binding and reduce organ overgrowth caused by Yap overexpression or Nf2 knockout[152]. A number of studies showed that VP could mimic YAP knockdown to overcome resistance to RAFi, TKI or chemo drugs [39,50,51,53,56,64,118,121,134,153]. However, Verteporfin only blocks YAP-TEAD binding at high micromolar concentrations and has YAP-independent effects and therefore is not a clinically viable YAP inhibitor $[152,154,155]$. A number of drugs were found to indirectly inhibit YAP/TAZ by targeting upstream regulators of Hippo signaling [156]. In particular, Statins, which target HMG-CoA reductase, inhibit YAP nuclear translocation and mimic genetic YAP ablation in sensitizing cells to TKI or MAPKi [52,53,60,157-159]. However, similar to VP, Statins exert pleiotropic cellular effects and require high concentrations to block YAP-mediated transcription $[157,158,160]$, rendering them unsuitable as clinical YAP inhibitors.

As an alternative to targeting upstream Hippo signaling, drugs that inhibit downstream effectors of YAP/TAZ showed promise in the clinic. For example, multiple small molecule AXL inhibitors (bemcentinib, TP-0903) and pamrevlumab (FG-3019) - a monoclonal CTGF antibody- are currently in clinical trials in combination with EGFRi and other cytoxic drugs $[161,162]$. However, given the diverse functions of YAP/TAZ targets, it is unlikely that targeting one downstream effector will fully recapitulate the effects of direct blockade of YAP/TAZ. Thus, it is important to further decipher the relative contributions of druggable YAP/TAZ targets to YAP/TAZ-mediated resistance to various types of cancer drugs (see outstanding questions). 
Notably, two recent studies reported that BET family protein BRD4 inhibitors (BRD4-i) could block YAP-mediated transcription and overcome resistance to MAPKi in BRAF mutant melanoma and KRAS mutant lung cancer cells $[38,62]$. Mechanistically, BRD4 was found to not only promote the expression of YAP but also directly interact with YAP/TAZ and function as a required co-factor for the YAP/TAZ/TEAD transcriptional complexes $[38,62]$. However, all the existing BRD4-i also inhibit BRD2 and BRD3, which have distinct functions and transcriptional targets [163]. Consequently, toxicity is a major concern for this type of drugs, and clinical utility is being investigated in trials of single agents or combinations with other agents [164]. Moreover, as a general transcription co-activator, BRD4 has numerous other transcriptional partners besides YAP/TAZ [163]. Conversely, YAP/TAZ are also known to partner with other epigenetic regulators to promote or suppress gene transcription [18,165-169]. Thus, the full extent of functional overlaps between YAP/TAZ and BRD4 in different cancer types represents another critical gap in knowledge (see outstanding questions).

Direct YAP/TAZ/TEAD inhibitors are currently under development [170]. Through function-based or in silico screenings, a number of lead compounds targeting various YAP/ TAZTEAD binding interfaces or various functional pockets of TEADs have been reported [170]. Furthermore, emerging evidence indicates that the activity of YAP/TAZ/TEAD transcriptional complexes are tightly controlled by extensive posttranslational modifications (PTMs) [122,137,171-177], an example of which is TEAD. Utilizing predicted 3D topography and solved crystal structures of TEAD interaction with many co-activators, TEAD homologs are shown to be consistently palmitoylated at an internal Cysteine and this modification is required for TEAD stability and binding propensity[175-177]. Due to significant functional overlaps between YAP and TEAD, targeting TEAD can potentially recapitulate full effects of YAP/TAZ inhibition [16-18]. However, It remains to be determined whether potent and specific small molecule inhibitors could be developed against critical PTM events, and whether targeting PTMs represents a viable approach to effectively block the activities of YAP/TAZ/TEAD transcription complexes (see outstanding questions).

While Hippo pathway's relevance to immunotherapy resistance has not been formally confirmed, the prospect is promising. Regulation of the expression of PD-L1 and other immune suppressive cytokines by YAP/TAZ implies that tumors can rely on Hippo signaling deregulation to evade immune surveillance, which further supports strategies targeting this pathway. However, it was reported that YAP/TAZ's regulation of PD-L1 transcription is human-specific[141], making it difficult to model this aspect of YAP/TAZ functions using the traditional syngenic or GEMM mouse models. Moreover, while YAP/TAZ are highly expressed across most solid tumor types, they are strongly silenced in blood malignancies $[178,179]$. Further complicating the matter is that Hippo-YAP/TAZ signaling in haematopoetic cells appears to be divergent in many aspects from non-haematopoetic cells and may be even different among different immune subtypes [180]. Thus, further studies are needed to fully anticipate systemic effect of targeting the Hippo-YAP/TAZ pathway, especially of the immune system (see outstanding questions). 


\section{ACKNOWLEDGEMENT}

We thank Shannon White for critical review of the manuscript. Work in Yi lab has received financial support from NIH (R01CA187090), Cancer Center Support Grant (CA051008), DF/HCC Kidney Cancer SPORE (CA101942), $\mathrm{V}$ foundation, Toulmin Pilot Award, Sher Grant and Advocure foundation.

We declare no financial/personal conflict of interest.

\section{REFERENCE}

1. Society AC (2018) Cancer Facts \& Figures 2018. Am. Cancer Soc DOI: 10.1182/ blood-2015-12-687814

2. Wu S et al. (2003) hippo encodes a Ste-20 family protein kinase that restricts cell proliferation and promotes apoptosis in conjunction with salvador and warts. Cell DOI: 10.1016/ S0092-8674(03)00549-X

3. Huang J et al. (2005) The Hippo signaling pathway coordinately regulates cell proliferation and apoptosis by inactivating Yorkie, the Drosophila homolog of YAP. Cell DOI: 10.1016/j.cell. 2005.06.007

4. Justice RW et al. (1995) The Drosophila tumor suppressor gene warts encodes a homolog of human myotonic dystrophy kinase and is required for the control of cell shape and proliferation. Genes Dev DOI: $10.1101 /$ gad.9.5.534

5. Tapon $\mathrm{N}$ et al. (2002) salvador promotes both cell cycle exit and apoptosis in Drosophila and is mutated in human cancer cell lines. Cell DOI: 10.1016/S0092-8674(02)00824-3

6. Harvey KF et al. (2003) The Drosophila Mst ortholog, hippo, restricts growth and cell proliferation and promotes apoptosis. Cell DOI: 10.1016/S0092-8674(03)00557-9

7. Meng Z et al. (2015) MAP4K family kinases act in parallel to MST1/2 to activate LATS $1 / 2$ in the Hippo pathway. Nat. Commun DOI: 10.1038/ncomms9357

8. Mo JS et al. (2015) Cellular energy stress induces AMPK-mediated regulation of YAP and the Hippo pathway. Nat. Cell Biol DOI: 10.1038/ncb3111

9. Sorrentino G et al. (2014) Metabolic control of YAP and TAZ by the mevalonate pathway. Nat. Cell Biol DOI: $10.1038 / \mathrm{ncb} 2936$

10. Feng X et al. (2014) Hippo-independent activation of YAP by the GNAQ uveal melanoma oncogene through a Trio-regulated Rho GTPase Signaling Circuitry. Cancer Cell DOI: 10.1016/ j.ccr.2014.04.016

11. Das A et al. (2016) YAP nuclear localization in the absence of cell-cell contact is mediated by a filamentous actin-dependent, Myosin IIand Phospho-YAP-independent pathway during extracellular matrix mechanosensing. J. Biol. Chem DOI: 10.1074/jbc.M115.708313

12. Li P et al. (2016) aE-catenin inhibits a Src-YAP1 oncogenic module that couples tyrosine kinases and the effector of hippo signaling pathway. Genes Dev DOI: 10.1101/gad.274951.115

13. Aragona M et al. (2013) A mechanical checkpoint controls multicellular growth through YAP/TAZ regulation by actin-processing factors. Cell DOI: 10.1016/j.cell.2013.07.042

14. Tang Y et al. (2016) Snail/Slug binding interactions with YAP/TAZ control skeletal stem cell selfrenewal and differentiation. Nat. Cell Biol DOI: 10.1038/ncb3394

15. Hong JH et al. (2005) TAZ, a transcriptional modulator of mesenchymal stem cell differentiation. Science (80-. ) DOI: 10.1126/science.1110955

16. Galli GG et al. (2015) YAP Drives Growth by Controlling Transcriptional Pause Release from Dynamic Enhancers. Mol. Cell DOI: 10.1016/j.molcel.2015.09.001

17. Zanconato $\mathrm{F}$ et al. (2015) Genome-wide association between YAP/TAZ/TEAD and AP-1 at enhancers drives oncogenic growth. Nat. Cell Biol DOI: 10.1038/ncb3216

18. Stein $\mathrm{C}$ et al. (2015) YAP1 Exerts Its Transcriptional Control via TEAD-Mediated Activation of Enhancers. PLoS Genet DOI: 10.1371/journal.pgen.1005465

19. Kantarjian HM et al. (2002) Imatinib mesylate (STI571) therapy for Philadelphia chromosomepositive chronic myelogenous leukemia in blast phase. Blood DOI: 10.1182/blood.V99.10.3547 
20. Kantarjian H et al. (2002) Hematologic and Cytogenetic Responses to Imatinib Mesylate in Chronic Myelogenous Leukemia. N. Engl. J. Med DOI: 10.1056/NEJMoa011573

21. Tsimberidou A-M (2015) TARGETED THERAPY IN CANCER HHS Public Access. Cancer Chemother Pharmacol DOI: 10.1007/s00280-015-2861-1

22. Long GV et al. (2011) Prognostic and clinicopathologic associations of oncogenic BRAF in metastatic melanoma. J. Clin. Oncol DOI: 10.1200/JCO.2010.32.4327

23. Sun C et al. (2014) Reversible and adaptive resistance to BRAF(V600E) inhibition in melanoma. Nature DOI: $10.1038 /$ nature13121

24. Villanueva $\mathbf{J}$ et al. (2010) Acquired Resistance to BRAF Inhibitors Mediated by a RAF Kinase Switch in Melanoma Can Be Overcome by Cotargeting MEK and IGF-1R/PI3K. Cancer Cell DOI: 10.1016/j.ccr.2010.11.023

25. Manzano JL et al. (2016) Resistant mechanisms to BRAF inhibitors in melanoma. Ann. Transl. Med DOI: 10.21037/atm.2016.06.07

26. Shalem O et al. (2014) Genome-scale CRISPR-Cas9 knockout screening in human cells. Science (80-. ) 343, 84-87 [PubMed: 24336571]

27. Zhang N et al. (2010) The Merlin/NF2 tumor suppressor functions through the YAP oncoprotein to regulate tissue homeostasis in mammals. Dev. Cell 19, 27-38 [PubMed: 20643348]

28. Yin F et al. (2013) Spatial organization of hippo signaling at the plasma membrane mediated by the tumor suppressor merlin/NF2. Cell 154, 1342-1355 [PubMed: 24012335]

29. Joung J et al. (2017) Genome-scale activation screen identifies a lncRNA locus regulating a gene neighbourhood. Nature 548, 343-346 [PubMed: 28792927]

30. Hwang J and Pallas DC (2014) STRIPAK complexes: Structure, biological function, and involvement in human diseases. Int. J. Biochem. Cell Biol 47, 118-148 [PubMed: 24333164]

31. Bae SJ et al. (2017) SAV1 promotes Hippo kinase activation through antagonizing the PP2A phosphatase STRIPAK. Elife 6,

32. Lin L et al. (2015) The Hippo effector YAP promotes resistance to RAF- and MEK-targeted cancer therapies. Nat. Genet 47, 250-256 [PubMed: 25665005]

33. Kim MH et al. (2016) Actin remodeling confers BRAF inhibitor resistance to melanoma cells through YAP/TAZ activation. EMBO J 35, 462-478 [PubMed: 26668268]

34. Yu FX et al. (2012) Regulation of the Hippo-YAP pathway by G-protein-coupled receptor signaling. Cell DOI: 10.1016/j.cell.2012.06.037

35. Prior IA et al. A comprehensive survey of ras mutations in cancer., Cancer Research (2012)

36. Kapoor A et al. (2014) Yap1 activation enables bypass of oncogenic KRAS addiction in pancreatic cancer. Cell 158, 185-197 [PubMed: 24954535]

37. Shao DD et al. (2014) KRAS and YAP1 converge to regulate EMT and tumor survival. Cell 158, 171-84 [PubMed: 24954536]

38. Kitajima S et al. (2018) Overcoming Resistance to Dual Innate Immune and MEK Inhibition Downstream of KRAS. Cancer Cell DOI: 10.1016/j.ccell.2018.08.009

39. Zhao X et al. (2017) A combinatorial strategy using YAP and pan-RAF inhibitors for treating KRAS-mutant pancreatic cancer. Cancer Lett DOI: 10.1016/j.canlet.2017.05.015

40. Sos ML et al. (2009) Identifying genotype-dependent efficacy of single and combined PI3K- and MAPK-pathway inhibition in cancer. Proc. Natl. Acad. Sci DOI: 10.1073/pnas.0907325106

41. She QB et al. (2010) 4E-BP1 Is a Key Effector of the Oncogenic Activation of the AKT and ERK Signaling Pathways that Integrates Their Function in Tumors. Cancer Cell DOI: 10.1016/j.ccr. 2010.05.023

42. Engelman JA et al. (2008) Effective use of PI3K and MEK inhibitors to treat mutant Kras G12D and PIK3CA H1047R murine lung cancers. Nat. Med DOI: 10.1038/nm.1890

43. Heist RS and et al. (2013), Combination of a MEK inhibitor, pimasertib (MSC1936369B), and a $\mathrm{PI} 3 \mathrm{~K} / \mathrm{mTOR}$ inhibitor, SAR245409, in patients with advanced solid tumors: Results of a phase Ib dose-escalation trial. , in ASCO

44. Bedard PL et al. (2015) A Phase Ib dose-escalation study of the oral pan-PI3K inhibitor buparlisib (BKM120) in combination with the oral MEK1/2 inhibitor trametinib (GSK1120212) in patients with selected advanced solid tumors. Clin. Cancer Res DOI: 10.1158/1078-0432.CCR-14-1814 
45. LoRusso P (2012) A first-in-human phase Ib study to evaluate the MEK inhibitor GDC-0973, combined with the pan-PI3K inhibitor GDC-0941, in patients with advanced solid tumors. J Clin Oncol DOI: $10.1200 /$ JCO.2012.42.1016

46. Hata AN et al. (2014) Failure to induce apoptosis via BCL-2 family proteins underlies lack of efficacy of combined MEK and PI3K inhibitors for KRAS-mutant lung cancers. Cancer Res DOI: 10.1158/0008-5472.CAN-13-3728

47. Chong CR and Jänne PA The quest to overcome resistance to EGFR-targeted therapies in cancer. , Nature Medicine (2013)

48. Kobayashi S et al. (2005) EGFR Mutation and Resistance of Non-Small-Cell Lung Cancer to Gefitinib. N. Engl. J. Med DOI: 10.1056/NEJMoa044238

49. Ghiso E et al. (2017) YAP-Dependent AXL Overexpression Mediates Resistance to EGFR Inhibitors in NSCLC. Neoplasia (United States) 19, 1012-1021

50. Lee JE et al. (2016) Hippo pathway effector YAP inhibition restores the sensitivity of EGFR-TKI in lung adenocarcinoma having primary or acquired EGFR-TKI resistance. Biochem. Biophys. Res. Commun 474, 154-160 [PubMed: 27105908]

51. Lin C-H et al. (2015) Microenvironment rigidity modulates responses to the HER2 receptor tyrosine kinase inhibitor lapatinib via YAP and TAZ transcription factors. Mol. Biol. Cell DOI: 10.1091/mbc.E15-07-0456

52. Zhou TY et al. (2016) Inactivation of hypoxia-induced YAP by statins overcomes hypoxic resistance tosorafenib in hepatocellular carcinoma cells article. Sci. Rep 6, 1-12 [PubMed: 28442746]

53. Lee TF et al. (2018) Enhanced YAP expression leads to EGFR TKI resistance in lung adenocarcinomas. Sci. Rep 8, 1-8 [PubMed: 29311619]

54. Guo L et al. (2016) Knockdown of TAZ modifies triple-negative breast cancer cell sensitivity to EGFR inhibitors by regulating YAP expression. Oncol. Rep 36, 729-736 [PubMed: 27373987]

55. Xu W et al. (2015) Up-regulation of the Hippo pathway effector TAZ renders lung adenocarcinoma cells harboring EGFR-T790M mutation resistant to gefitinib. Cell Biosci 5, 1-9 [PubMed: 25601894]

56. Hsu P et al. (2016) YAP promotes erlotinib resistance in human non-small cell lung cancer cells. Oncotarget 7 ,

57. Wang H et al. (2016) Tankyrase inhibitor sensitizes lung cancer cells to endothelial growth factor receptor (EGFR) inhibition via stabilizing angiomotins and inhibiting yap signaling. J. Biol. Chem 291, 15256-15266 [PubMed: 27231341]

58. Lee WY et al. (2017) Panobinostat sensitizes KRAS-mutant non-small-cell lung cancer to gefitinib by targeting TAZ. Int. J. Cancer 141, 1921-1931 [PubMed: 28710768]

59. Mcgowan M et al. (2017) NSCLC depend upon YAP expression and nuclear localization after acquiring resistance to EGFR inhibitors. Genes Cancer 8,

60. Liu B-S et al. (2018) Inhibition of YAP reverses primary resistance to EGFR inhibitors in colorectal cancer cells. Oncol. Rep 40, 2171-2182 [PubMed: 30106444]

61. Xu MZ et al. (2011) AXL receptor kinase is a mediator of YAP-dependent oncogenic functions in hepatocellular carcinoma. Oncogene DOI: 10.1038/onc.2010.504

62. Zanconato $\mathrm{F}$ et al. (2018) Transcriptional addiction in cancer cells is mediated by YAP/TAZ through BRD4. Nat. Med DOI: 10.1038/s41591-018-0158-8

63. Zuo Q et al. (2018) AXL/AKT axis mediated-resistance to BRAF inhibitor depends on PTEN status in melanoma. Oncogene DOI: 10.1038/s41388-018-0205-4

64. Song S et al. (2015) The hippo coactivator YAP1 mediates EGFR overexpression and confers chemoresistance in esophageal cancer. Clin. Cancer Res 21, 2580-2590 [PubMed: 25739674]

65. Lehmann W et al. (2016) ZEB1 turns into a transcriptional activator by interacting with YAP1 in aggressive cancer types. Nat. Commun 7, 1-15

66. Song Y et al. (2017) TGF- $\beta$-independent CTGF induction regulates cell adhesion mediated drug resistance by increasing collagen I in HCC. Oncotarget DOI: 10.18632/oncotarget.15521

67. Muranen T et al. (2016) ERK and p38 MAPK activities determine sensitivity to PI3K/mTOR inhibition via regulation of MYC and YAP. Cancer Res 76, 7168-7180 [PubMed: 27913436] 
68. Oku Y et al. (2018) Augmentation of the therapeutic efficacy of WEE1 kinase inhibitor AZD1775 by inhibiting the YAP-E2F1-DNA damage response pathway axis. FEBS Open Bio DOI: $10.1002 / 2211-5463.12440$

69. OKU Y et al. (2018) Sensitisation of Cancer Cells to MLN8237, an Aurora-A Inhibitor, by YAP/TAZ Inactivation. Anticancer Res DOI: 10.21873/anticanres.12617

70. Li Z et al. (2018) Loss of the FAT1 Tumor Suppressor Promotes Resistance to CDK4/6 Inhibitors via the Hippo Pathway. Cancer Cell 34, 893-905.e8 [PubMed: 30537512]

71. Chorzalska A et al. (2017) Long-Term Exposure to Imatinib Mesylate Downregulates Hippo Pathway and Activates YAP in a Model of Chronic Myelogenous Leukemia. Stem Cells Dev DOI: 10.1089/scd.2016.0262

72. Perez EA (2009) Microtubule inhibitors: Differentiating tubulin-inhibiting agents based on mechanisms of action, clinical activity, and resistance. Mol. Cancer Ther DOI: 10.1158/1535-7163.MCT-09-0366

73. Rowinsky EK (1997) THE DEVELOPMENT AND CLINICAL UTILITY OF THE TAXANE CLASS OF ANTIMICROTUBULE CHEMOTHERAPY AGENTS,

74. Ji D et al. (2007) A screen of shRNAs targeting tumor suppressor genes to identify factors involved in A549 paclitaxel sensitivity. Oncol. Rep

75. Zhao Y et al. (2014) YAP-induced resistance of cancer cells to antitubulin drugs is modulated by a hippo-independent pathway. Cancer Res 74, 4493-4503 [PubMed: 24812269]

76. Lai D et al. (2011) Taxol resistance in breast cancer cells is mediated by the hippo pathway component TAZ and its downstream transcriptional targets Cyr61 and CTGF. Cancer Res 71, 2728-2738 [PubMed: 21349946]

77. Cordenonsi M et al. (2011) The hippo transducer TAZ confers cancer stem cell-related traits on breast cancer cells. Cell 147, 759-772 [PubMed: 22078877]

78. Yang S et al. (2013) CDK1 phosphorylation of YAP promotes mitotic defects and cell motility and is essential for neoplastic transformation. Cancer Res 73, 6722-6733 [PubMed: 24101154]

79. Jeong W et al. (2014) Activation of YAP1 is associated with poor prognosis and response to taxanes in ovarian cancer. Anticancer Res

80. Yeung B et al. (2018) Identification of Cdk1-LATS-Pin1 as a novel signaling axis in anti-tubulin drug response of cancer cells. Mol. Cancer Res DOI: 10.1158/1541-7786.MCR-17-0684

81. Mohamed Z et al. (2018) miR-363 confers taxane resistance in ovarian cancer by targeting the Hippo pathway member, LATS2. Oncotarget 9, 30053-30065 [PubMed: 30046387]

82. Xia Y et al. (2014) YAP/TEAD Co-Activator regulated pluripotency and chemoresistance in ovarian cancer Initiated Cells. PLoS One 9,

83. Fujimoto D et al. (2015) PAR1 participates in the ability of multidrug resistance and tumorigenesis by controlling Hippo-YAP pathway. Oncotarget 6, 34788-34799 [PubMed: 26431277]

84. Zhao Y and Yang X (2015) Regulation of sensitivity of tumor cells to antitubulin drugs by Cdk1TAZ signalling. Oncotarget 6, 21906-21917 [PubMed: 26183396]

85. Chen $\mathrm{M}$ et al. (2015) Upregulation of miR-181c contributes to chemoresistance in pancreatic cancer by inactivating the Hippo signaling pathway. Oncotarget 6, 44466-44479 [PubMed: 26561204]

86. Castedo $\mathrm{M}$ et al. Cyclin-dependent kinase-1: Linking apoptosis to cell cycle and mitotic catastrophe. , Cell Death and Differentiation (2002)

87. Nakayama $\mathrm{S}$ et al. (2009) Prediction of paclitaxel sensitivity by CDK1 and CDK2 activity in human breast cancer cells. Breast Cancer Res DOI: 10.1186/bcr2231

88. Chen M et al. (2018) miR-590-5p suppresses hepatocellular carcinoma chemoresistance by targeting YAP1 expression. EBioMedicine 35, 142-154 [PubMed: 30111512]

89. Wang MY et al. (2009) Connective tissue growth factor confers drug resistance in breast cancer through concomitant up-regulation of Bcl-xL and clap1. Cancer Res 69, 3482-3491 [PubMed: 19351859]

90. Menendez JA et al. (2005) A novel CYR61-triggered "CYR61-avß3integrin loop" regulates breast cancer cell survival and chemosensitivity through activation of ERK1/ERK2 MAPK signaling pathway. Oncogene 24, 761-779 [PubMed: 15592521] 
91. Tsai HC et al. (2014) CTGF increases drug resistance to paclitaxel by upregulating survivin expression in human osteosarcoma cells. Biochim. Biophys. Acta - Mol. Cell Res 1843, 846-854

92. Lin M et al. (2004) Cyr61 Expression Confers Resistance to Apoptosis in Breast Cancer MCF-7 Cells by a Mechanism of NF- $\kappa$ B-dependent XIAP Up-Regulation. J. Biol. Chem 279, 2401524023 [PubMed: 15044484]

93. Chang AY et al. (2007) Phase II trial of 5-fluorouracil/leucovorin/gemcitabine/cisplatin as secondline treatment in patients with metastatic or recurrent colorectal carcinoma: A cancer therapeutics research group study. Clin. Colorectal Cancer DOI: 10.3816/CCC.2007.n.033

94. Amrutkar M and Gladhaug IP Pancreatic cancer chemoresistance to gemcitabine. , Cancers (2017)

95. Telli ML et al. (2015) Phase II study of gemcitabine, carboplatin, and iniparib as neoadjuvant therapy for triple-negative and BRCA1/2 mutation-associated breast cancer with assessment of a tumor-based measure of genomic instability: PrECOG 0105. J. Clin. Oncol DOI: 10.1200/JCO. 2014.57.0085

96. Moore DF et al. (1992) Phase II study of gemcitabine in advanced colorectal adenocarcinoma. Invest. New Drugs DOI: 10.1007/BF00944189

97. Jia Y and Xie J Promising molecular mechanisms responsible for gemcitabine resistance in cancer., Genes and Diseases (2015)

98. Binenbaum Y et al. (2015) Gemcitabine resistance in pancreatic ductal adenocarcinoma. Drug Resist. Updat DOI: 10.1016/j.drup.2015.10.002

99. Longley DB et al. 5-Fluorouracil: Mechanisms of action and clinical strategies. , Nature Reviews Cancer (2003)

100. Gonen N and Assaraf YG Antifolates in cancer therapy: Structure, activity and mechanisms of drug resistance. , Drug Resistance Updates (2012)

101. Zhan T et al. (2018) Downregulation of MicroRNA-455-3p Links to Proliferation and Drug Resistance of Pancreatic Cancer Cells via Targeting TAZ. Mol. Ther. - Nucleic Acids 10, 215 226 [PubMed: 29499934]

102. Que K et al. (2017) Downregulation of miR-874-3p promotes chemotherapeutic resistance in colorectal cancer via inactivation of the Hippo signaling pathway. Oncol. Rep 38, 3376-3386 [PubMed: 29039607]

103. Song R et al. (2018) Functional significance of Hippo/YAP signaling for drug resistance in colorectal cancer. Mol. Carcinog 57, 1608-1615 [PubMed: 30074279]

104. Overholtzer M et al. (2006) Transforming properties of YAP, a candidate oncogene on the chromosome 11q22 amplicon. Proc. Natl. Acad. Sci 103, 12405-12410 [PubMed: 16894141]

105. Yuan L et al. (2017) Palmitic acid dysregulates the Hippo-YAP pathway and inhibits angiogenesis by inducing mitochondrial damage and activating the cytosolic DNA sensor cGASSTING-IRF3 signaling mechanism DOI: 10.1074/jbc.M117.804005

106. Tian Y et al. (2016) Metformin mediates resensitivity to 5-fluorouracil in hepatocellular carcinoma via the suppression of YAP. Oncotarget 7, 46230-46241 [PubMed: 27323827]

107. Gujral TS and Kirschner MW (2017) Hippo pathway mediates resistance to cytotoxic drugs. Proc. Natl. Acad. Sci 114, E3729-E3738 [PubMed: 28416665]

108. Ciamporcero E et al. (2018) Crosstalk between Nrf2 and YAP contributes to maintaining the antioxidant potential and chemoresistance in bladder cancer. Free Radic. Biol. Med DOI: 10.1016/j.freeradbiomed.2017.12.005

109. Gao ZQ et al. (2018) Long non-coding RNA GAS5 antagonizes the chemoresistance of pancreatic cancer cells through down-regulation of miR-181c-5p. Biomed. Pharmacother 97, 809-817 [PubMed: 29112934]

110. Wang DY et al. (2016) Hippo/YAP signaling pathway is involved in osteosarcoma chemoresistance. Chin. J. Cancer 35, 47 [PubMed: 27206784]

111. Yang K and Lin C (2016) CTGF enhances resistance to 5-FU-mediating cell apoptosis through FAK/MEK/ERK signal pathway in colorectal cancer. Oncotarget 9, 7285-7295

112. Cox AG et al. (2018) Yap regulates glucose utilization and sustains nucleotide synthesis to enable organ growth DOI: $10.15252 / \mathrm{embj} .2018100294$ 
113. Wang W et al. (2015) AMPK modulates Hippo pathway activity to regulate energy homeostasis. Nat. Cell Biol 17, 490-499 [PubMed: 25751139]

114. Edwards DN et al. (2017) The receptor tyrosine kinase EphA2 promotes glutamine metabolism in tumors by activating the transcriptional coactivators YAP and TAZ. Sci. Signal 10, eaan4667 [PubMed: 29208682]

115. Cosset É et al. (2017) Glut3 Addiction Is a Druggable Vulnerability for a Molecularly Defined Subpopulation of Glioblastoma. Cancer Cell 32, 856-868.e5 [PubMed: 29198914]

116. Liu P et al. (2017) Central role of mTORC1 downstream of YAP/TAZ in hepatoblastoma development. Oncotarget 8, 73433-73447 [PubMed: 29088718]

117. Dai X et al. (2016) Nuclear translocation and activation of YAP by hypoxia contributes to the chemoresistance of SN38 in hepatocellular carcinoma cells. Oncotarget 7,

118. Dai Y et al. (2017) YAP1 regulates ABCG2 and cancer cell side population in human lung cancer cells. Oncotarget 8, 4096-4109 [PubMed: 27911857]

119. Huo X et al. (2013) Overexpression of Yes-associated protein confers doxorubicin resistance in hepatocellullar carcinoma. Oncol. Rep 29, 840-846 [PubMed: 23232767]

120. Lu T et al. (2018) Yes-associated protein enhances proliferation and attenuates sensitivity to cisplatin in human gastric cancer cells. Biomed. Pharmacother 105, 1269-1275 [PubMed: 30021363]

121. Ma K et al. (2016) Nuclear accumulation of Yes-Associated Protein (YAP) maintains the survival of doxorubicin-induced senescent cells by promoting survivin expression. Cancer Lett DOI: 10.1016/j.canlet.2016.02.045

122. Mao B et al. (2014) SIRT1 regulates YAP2-mediated cell proliferation and chemoresistance in hepatocellular carcinoma. Oncogene 33, 1468-1474 [PubMed: 23542177]

123. Ren A et al. (2008) Down-regulation of mammalian sterile 20-like kinase 1 by heat shock protein 70 mediates cisplatin resistance in prostate cancer cells. Cancer Res 68, 2266-2274 [PubMed: 18381433]

124. Song J et al. (2018) Role of YAP in lung cancer resistance to cisplatin. Oncol. Lett 16, 3949-3954 [PubMed: 30128013]

125. Tian T et al. (2015) TAZ promotes temozolomide resistance by upregulating MCL-1 in human glioma cells. Biochem. Biophys. Res. Commun 463, 638-643 [PubMed: 26043698]

126. Xiao L et al. (2016) YAP induces cisplatin resistance through activation of autophagy in human ovarian carcinoma cells. Onco. Targets. Ther 9, 1105-14 [PubMed: 27073322]

127. Wang X et al. (2018) Downregulation of YAP inhibits proliferation, invasion and increases cisplatin sensitivity in human hepatocellular carcinoma cells. Oncol. Lett 16, 585-593 [PubMed: 29928445]

128. $\mathrm{Xu} \mathrm{W}$ et al. (2017) TAZ inhibition restores sensitivity of cisplatin via AKT/mTOR signaling in lung adenocarcinoma. Oncol. Rep DOI: 10.3892/or.2017.5847

129. Xu M et al. (2018) MiR-149-5p promotes chemotherapeutic resistance in ovarian cancer via the inactivation of the Hippo signaling pathway. Int. J. Oncol 52, 815-827 [PubMed: 29393390]

130. Yang $\mathrm{C}$ et al. (2017) YAP promotes tumorigenesis and cisplatin resistance in neuroblastoma. Oncotarget 8, 1-10 [PubMed: 27980222]

131. Yoshikawa K et al. (2015) The Hippo pathway transcriptional co-activator, YAP, confers resistance to cisplatin in human oral squamous cell carcinoma. Int. J. Oncol 46, 2364-2370 [PubMed: 25846049]

132. Bi L et al. (2017), AJUBA increases the cisplatin resistance through hippo pathway in cervical cancer. , Gene, 644, Elsevier, 148-154 [PubMed: 29126926]

133. Bartucci $\mathrm{M}$ et al. (2015) TAZ is required for metastatic activity and chemoresistance of breast cancer stem cells. Oncogene 34, 681-690 [PubMed: 24531710]

134. Ciamporcero E et al. (2016) YAP activation protects urothelial cell carcinoma from treatmentinduced DNA damage. Oncogene 35, 1541-1553 [PubMed: 26119935]

135. Zeng $\mathrm{H}$ et al. (2017) Connective tissue growth factor promotes temozolomide resistance in glioblastoma through TGF- $\beta 1$-dependent activation of Smad/ERK signaling. Cell Death Dis 8, 1-12 [PubMed: 29233966] 
136. Yin D et al. (2010) Connective tissue growth factor associated with oncogenic activities and drug resistance in glioblastoma multiforme. Int. J. cancer 127, 2257-67 [PubMed: 20162579]

137. Hata $\mathrm{S}$ et al. (2012) A novel acetylation cycle of transcription co-activator yes-associated protein that is downstream of hippo pathway is triggered in response to S N2 alkylating agents. J. Biol. Chem DOI: 10.1074/jbc.M111.334714

138. Pitt JM et al. Resistance Mechanisms to Immune-Checkpoint Blockade in Cancer: TumorIntrinsic and -Extrinsic Factors. , Immunity (2016)

139. Restifo NP et al. Acquired resistance to immunotherapy and future challenges., Nature Reviews Cancer (2016)

140. Sharma P et al. Primary, Adaptive, and Acquired Resistance to Cancer Immunotherapy. , Cell (2017)

141. Helena JJ van R et al. (2018) The hippo pathway component taz promotes immune evasion in human cancer through PD-L1. Cancer Res 78, 1457-1470 [PubMed: 29339539]

142. Hsu PC et al. (2018) Inhibition of yes-associated protein down-regulates PD-L1 (CD274) expression in human malignant pleural mesothelioma. J. Cell. Mol. Med 22, 3139-3148 [PubMed: 29575535]

143. Kim MH et al. (2018) YAP-induced PD-L1 expression drives immune evasion in BRAFi-resistant melanoma. Cancer Immunol. Res DOI: 10.1158/2326-6066.CIR-17-0320

144. Janse van Rensburg HJ et al. (2018) The Hippo Pathway Component TAZ Promotes Immune Evasion in Human Cancer through PD-L1. Cancer Res 78, 1457-1470 [PubMed: 29339539]

145. Lee BS et al. (2017) Hippo effector YAP directly regulates the expression of PD-L1 transcripts in EGFR-TKI-resistant lung adenocarcinoma. Biochem. Biophys. Res. Commun 491, 493-499 [PubMed: 28684311]

146. Miao J et al. (2017) YAP regulates PD-L1 expression in human NSCLC cells. Oncotarget 8, 114576-114587 [PubMed: 29383103]

147. Lindau D et al. (2012) The immunosuppressive tumour network: myeloid-derived suppressor cells, regulatory T cells and natural killer T cells. Immunology DOI: 10.1111/imm. 12036

148. Wang G et al. (2016) Targeting YAP-dependent MDSC infiltration impairs tumor progres1. Wang G, Lu X, Dey P, et al. Targeting YAP-dependent MDSC infiltration impairs tumor progression. Cancer Discov 2016;6(1):80-95. doi:10.1158/2159-8290.CD-15-0224sion. Cancer Discov. 6, 8095 [PubMed: 26701088]

149. Murakami S et al. (2017) Yes-associated protein mediates immune reprogramming in pancreatic ductal adenocarcinoma. Oncogene 36, 1232-1244 [PubMed: 27546622]

150. Kim W et al. (2017), Hepatic Hippo signaling inhibits protumoural microenvironment to suppress hepatocellular carcinoma. , Gut

151. Guo X et al. (2017) Single tumor-initiating cells evade immune clearance by recruiting type II macrophages. Genes Dev 31, 247-259 [PubMed: 28223311]

152. Liu-Chittenden Y et al. (2012) Genetic and pharmacological disruption of the TEADYAP complex suppresses the oncogenic activity of YAP. Genes Dev 26, 1300-1305 [PubMed: 22677547]

153. Fisher ML et al. (2017) Inhibition of YAP function overcomes BRAF inhibitor resistance in melanoma cancer stem cells. Oncotarget 8, 110257-110272 [PubMed: 29299145]

154. Fortin J et al. (2013) Verteporfin exhibits YAP-independent anti-proliferative and cytotoxic effects in endometrial cancer cells. Endocrinology DOI: 10.18632/oncotarget.15614

155. Donohue E et al. (2011) Inhibition of autophagosome formation by the benzoporphyrin derivative verteporfin. J. Biol. Chem DOI: 10.1074/jbc.M110.139915

156. Tao Y et al. (2017) The Hippo signaling pathway: An emerging anti-cancer drug target. Discov. Med

157. Sorrentino G et al. (2014) Metabolic control of YAP and TAZ by the mevalonate pathway. Nat. Cell Biol 16, 357-366 [PubMed: 24658687]

158. Oku Y et al. (2015) Small molecules inhibiting the nuclear localization of YAP/TAZ for chemotherapeutics and chemosensitizers against breast cancers. FEBS Open Bio DOI: 10.1016/ j.fob.2015.06.007 
159. Xia H et al. (2018) EGFR-PI3K-PDK1 pathway regulates YAP signaling in hepatocellular carcinoma: The mechanism and its implications in targeted therapy article. Cell Death Dis 9,

160. Matusewicz L et al. The effect of statins on cancer cells-review. , Tumor Biology (2015)

161. Gay CM et al. Giving AXL the axe: Targeting AXL in human malignancy., British Journal of Cancer (2017)

162. Heestand GM et al. (2011) A phase I trial of the monoclonal antibody FG-3019 to connective tissue growth factor (CTGF) in locally advanced or metastatic pancreatic cancer. J. Clin. Oncol

163. Xu Y and Vakoc CR (2017) Targeting cancer cells with BET bromodomain inhibitors. Cold Spring Harb. Perspect. Med DOI: 10.1101/cshperspect.a026674

164. Andrieu $\mathrm{G}$ et al. Clinical trials for BET inhibitors run ahead of the science. , Drug Discovery Today: Technologies (2016)

165. Skibinski A et al. (2014) The Hippo Transducer TAZ Interacts with the SWI/SNF Complex to Regulate Breast Epithelial Lineage Commitment. Cell Rep DOI: 10.1016/j.celrep.2014.02.038

166. Oh H et al. (2014) Yorkie Promotes Transcription by Recruiting a Histone Methyltransferase Complex. Cell Rep DOI: 10.1016/j.celrep.2014.06.017

167. Oh H et al. (2013) Genome-wide Association of Yorkie with Chromatin and ChromatinRemodeling Complexes. Cell Rep DOI: 10.1016/j.celrep.2013.01.008

168. Kim M et al. (2015) Transcriptional co-repressor function of the hippo pathway transducers YAP and TAZ. Cell Rep DOI: 10.1016/j.celrep.2015.03.015

169. Elster D et al. (2018) TRPS1 shapes YAP/TEAD-dependent transcription in breast cancer cells. Nat. Commun DOI: 10.1038/s41467-018-05370-7

170. Gibault F et al. (2018) Targeting Transcriptional Enhanced Associate Domains (TEADs). J. Med. Chem DOI: 10.1021/acs.jmedchem.7b00879

171. Peng C et al. (2017) Regulation of the Hippo-YAP Pathway by Glucose Sensor OGlcNAcylation. Mol. Cell 68, 591-604.e5 [PubMed: 29100056]

172. Zhang X et al. (2017) The essential role of YAP O-GlcNAcylation in high-glucose-stimulated liver tumorigenesis. Nat. Commun 8, 15280 [PubMed: 28474680]

173. Fang L et al. (2018) SET1A-Mediated Mono-Methylation at K342 Regulates YAP Activation by Blocking Its Nuclear Export and Promotes Tumorigenesis. Cancer Cell DOI: 10.1016/j.ccell. 2018.06.002

174. Gandhirajan RK et al. (2016) Cysteine S-glutathionylation promotes stability and activation of the Hippo downstream effector transcriptional Co-activator with PDZ-binding motif (TAZ). J. Biol. Chem DOI: 10.1074/jbc.M115.712539

175. Chan P et al. (2016) Autopalmitoylation of TEAD proteins regulates transcriptional output of the Hippo pathway. Nat. Chem. Biol DOI: 10.1038/nchembio.2036

176. Noland CL et al. (2016) Palmitoylation of TEAD Transcription Factors Is Required for Their Stability and Function in Hippo Pathway Signaling. Structure DOI: 10.1016/j.str.2015.11.005

177. Mesrouze Y et al. (2017) Effect of the acylation of TEAD4 on its interaction with co-activators YAP and TAZ. Protein Sci DOI: 10.1002/pro.3312

178. Levy D et al. (2008) Yap1 Phosphorylation by c-Abl Is a Critical Step in Selective Activation of Proapoptotic Genes in Response to DNA Damage. Mol. Cell DOI: 10.1016/j.molcel.2007.12.022

179. Cottini F et al. (2014) Rescue of Hippo coactivator YAP1 triggers DNA damage-induced apoptosis in hematological cancers. Nat. Med 20, 599-606 [PubMed: 24813251]

180. Hong L et al. (2018) Role of Hippo signaling in regulating immunity. Cell. Mol. Immunol 15, 1003-1009 [PubMed: 29568120] 


\section{Highlights}

- Hippo-YAP/TAZ signaling pathway has emerged as a linchpin in targeted-, chemo-, and potentially immune-therapy resistance

- In response to various therapies, numerous upstream signals could impinge on various components of the Hippo pathway to activate YAP/TAZ

- $\quad$ YAP/TAZ activation represents a central bypass mechanism to EGFR and RAS/MAPK blockade

- $\quad$ YAP/TAZ promote immune suppression by transcribing PD-L1 and cytokines required for polarization and recruitment of MDSCs

- Inhibitors targeting key YAP/TAZ transcriptional targets such as CTGF, AXL and Bcl-xl are under clinical trials in combination with standard therapies

- $\quad$ BET inhibitors have emerged as proxy inhibitors for YAP/TAZ 
- $\quad$ Given YAP/TAZ's roles in transcribing a diverse set of target genes, what are relative contributions of druggable YAP/TAZ targets to YAP/TAZ-mediated resistance to various cancer drugs in different cancer subtypes?

- Given the impact of PTMs on the folding, stability, subcellular localization, and binding affinities of YAP, TAZ and TEAD proteins, could small molecular inhibitors be designed to block PTMs that are essential for the activities of YAP/TAZ/TEAD?

- Given that BRD4 has recently emerged as a critical co-activator of the YAP/TAZ/TEAD transcriptional complexes, could BET inhibitors fully recapitulate the effects of YAP/TAZ blockade?

- What are the broader impacts of YAP/TAZ on the tumor microenvironment, particularly of the immune system? 


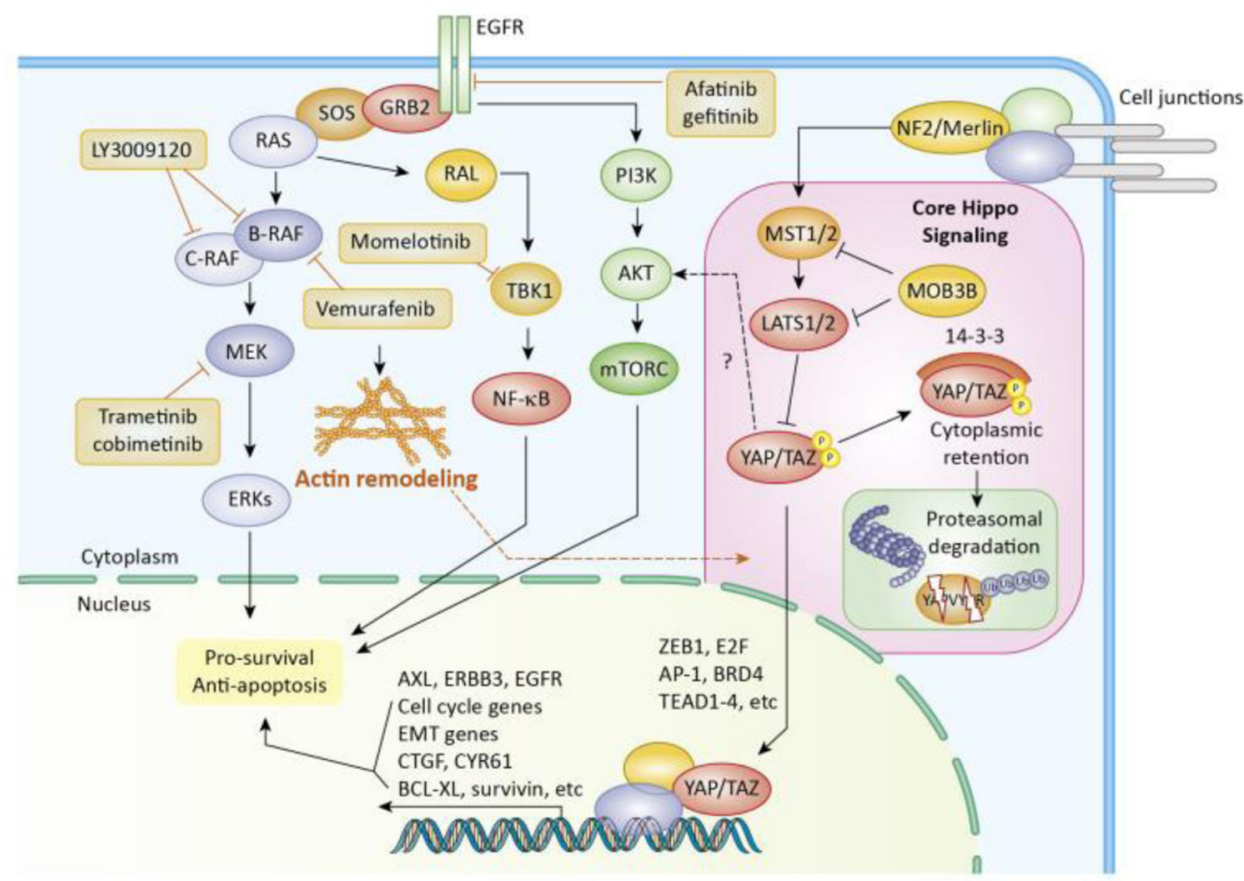

Figure 1. YAP/TAZ signaling and resistance to targeted therapy.

The Hippo pathway, which consists of a core MST1/2-LATS1/2 kinase cassette and numerous regulatory proteins including NF2/Merlin, SAV1, MOB1, MOB3B,

phosphorylates and inhibits YAP/TAZ by targeting them for cytoplasmic retention and proteasomal degradation mediated by $14-3-3$ and $\beta$-TrCP, respectively. Inactivation of Hippo signaling promotes stabilization and nuclear entry of YAP/TAZ, where they form transcription complexes with TEAD1-4, AP-1, BRD4, ZEB1, E2F and possibly other transcription factors to induce the expression of pro-survival/anti-apoptotic genes such as CTGF, CYR61, Bcl-xL and Survivin. YAP/TAZ have been identified as major drivers of resistance to RAF and MEK inhibitors as single agents or in combination with TBK1 inhibitor in BRAF and KRAS mutant cancer cells. BRAFi treatment induces actin cytoskeletal remodeling and promotes Rho-mediated YAP nuclear entry in BRAF-mutant melanoma cells. YAP induces E2F and AP-1-mediated cell cycle and EMT transcriptional programs to promote survival following ablation of oncogenic Kras in pancreatic and colon cancer cells. Additionally, YAP/TAZ induce EGFRi resistance by increasing the expression of AXL, ERBB3 and EGFR. 


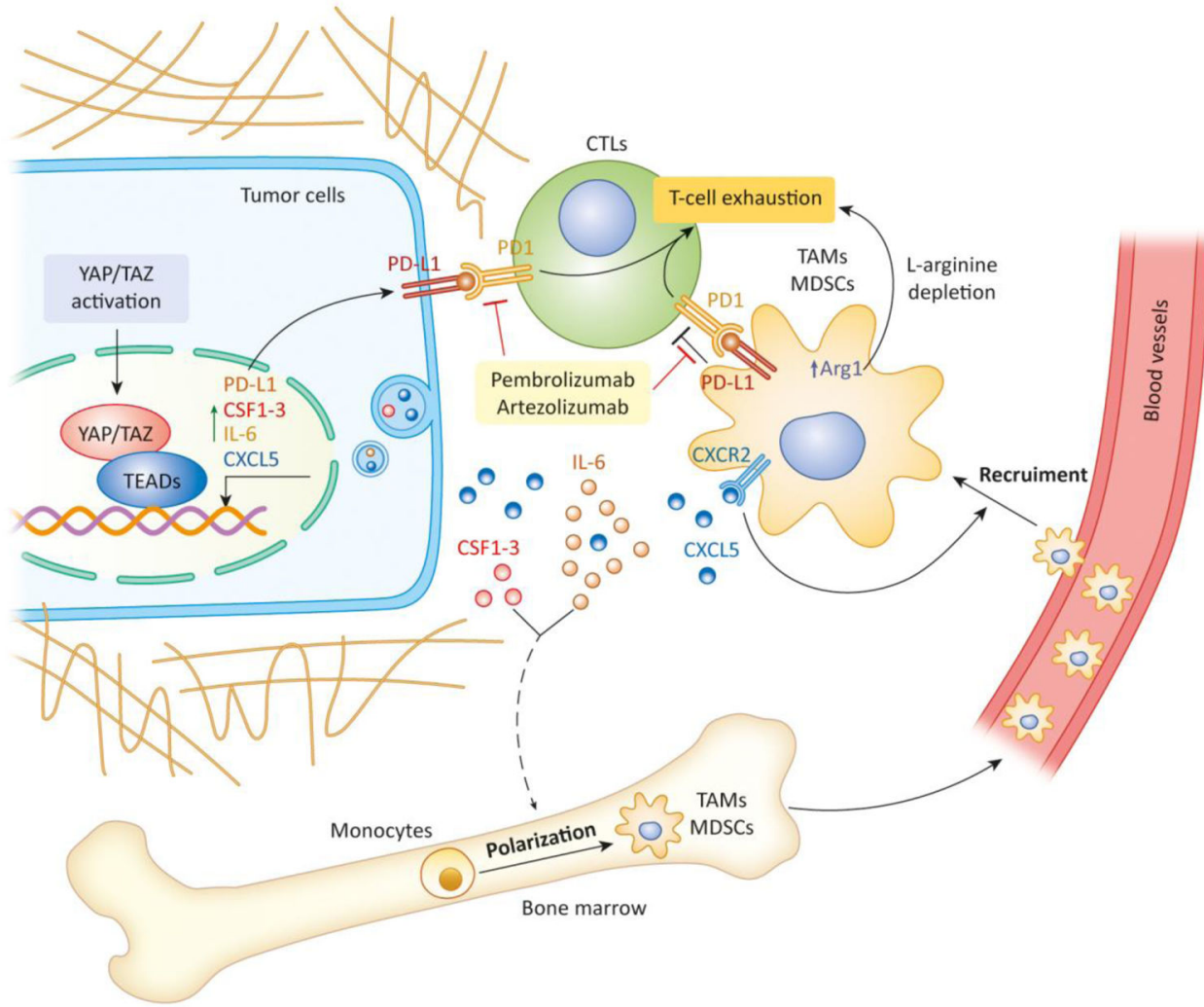

Figure 2. YAP/TAZ signaling and resistance to chemotherapy.

Multiple miRNAs are up- or down-regulated in response to various chemo drugs and induce chemo resistance by targeting different components of the Hippo pathway (MST1, SAV1, LATS2, MOB1, YAP) in various cancer types. Anti-microtubule treatment activates CDK1, which phosphorylates and promotes LATS binding to and inhibition of Pin1, an oncoprotein that induces taxane resistance in multiple malignancies. Furthermore, CDK1 directly phosphorylates and inhibits YAP/TAZ by disrupting their binding to TEADs and destabilizing TAZ, sensitizing tumor cells to anti-microtubule drugs. The transcriptional activity of YAP is further modulated by CBP/p300-mediated acetylation and SIRT1mediated deacetylation in response to various chemo agents. YAP/TAZ induce the transcription of antiapoptotic proteins (Bcl-xL, IAP1, Survivin.) and ABC multi-drug transporters to promote tumor cell survival and drug efflux, respectively. In addition, CTGF and CYR61, two canonical transcription targets of YAP/TAZ, can act through integrin receptors to activate MAPK and NF- $\mathrm{BB}$ signaling to further boost chemotherapy resistance. 


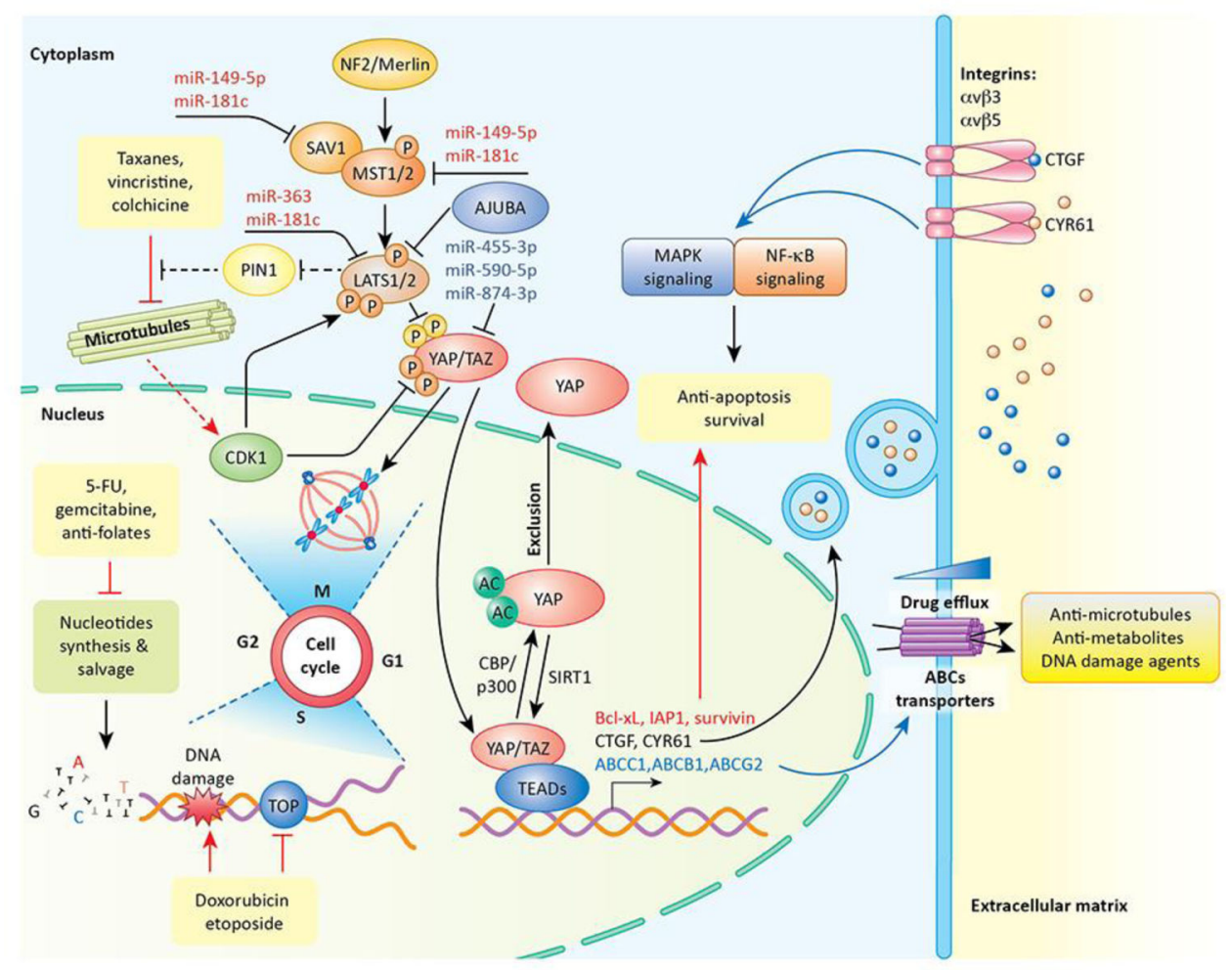

Figure 3. YAP/TAZ signaling and resistance to immune checkpoint therapy.

YAP/TAZ/TEAD directly transcribe PD-L1 and multiple cytokines (CSF1-3, IL6, CXCL5). YAP/TAZ-mediated PD-L1 expression on tumor cells binds to PD-1 expressed on effector T cells (CTLs), leading to inactivation of CTLs. Furthermore, YAP-induced upregulation of IL-6 and CSF1-3 skew the monocyte polarization in favor of tumor-promoting MDSCs and TAMs in bone marrow. On the other hand, YAP-mediated expression of CXCL5 by tumor cells attracts MDSCs and TAMs through its cognate receptor CXCR2 expressed on MDSCs and TAMs. Once recruited into the tumor microenvironment, MDSCs and TAMs inactivate CTLs both directly via PD-L1-PD interaction and indirectly by upregulating Arg1 and various other mechanisms. 